\title{
The metabolism of cancer cells during metastasis
}

\author{
Gabriele Bergers ${ }^{1,2}$ and Sarah-Maria Fendt ${ }^{3,4}$ \\ ${ }^{1}$ Laboratory of Tumor Microenvironment and Therapeutic Resistance, VIB-KU Leuven Center for Cancer Biology, Department of \\ Oncology, KU Leuven, Herestraat 49, 3000 Leuven, Belgium \\ ${ }^{2}$ UCSF Comprehensive Cancer Center, Department of Neurological Surgery, UCSF, San Francisco, CA, US \\ ${ }^{3}$ Laboratory of Cellular Metabolism and Metabolic Regulation, VIB-KU Leuven Center for Cancer Biology, VIB, Herestraat 49, 3000 \\ Leuven, Belgium \\ ${ }^{4}$ Laboratory of Cellular Metabolism and Metabolic Regulation, Department of Oncology, KU Leuven and Leuven Cancer Institute (LKI), \\ Herestraat 49, 3000 Leuven, Belgium \\ *email: S.-M.F., sarah-maria.fendt@kuleuven.vib.be; G.B., gabriele.bergers@kuleuven.vib.be
}

\begin{abstract}
Metastasis formation is the major cause of death in most patients with cancer. Despite extensive research, targeting metastatic seeding and colonization is still an unresolved challenge. Only recently, attention has been drawn to the fact that metastasizing cancer cells selectively and dynamically adapt their metabolism at every step during the metastatic cascade. Moreover, many metastases display different metabolic traits compared with the tumours from which they originate, enabling survival and growth in the new environment. Consequently, the stage-dependent metabolic traits may provide therapeutic windows for preventing or reducing metastasis, and targeting the new metabolic traits arising in established metastases may allow their eradication.
\end{abstract}

The metastatic establishment of cancers at distant organs is largely uncurable and primarily contributes to the deaths of cancer patients. Nonetheless, metastasis for- mation itself is a rare event in tumours because cancer cells need to overcome multiple environmental hur- dles before they can successfully manifest themselves in other organs ${ }^{1,2}$. As a first step, cancer cells need to become motile, invasive and intravasate the tumour vasculature to enter the bloodstream, either directly or via the lymphatic system. Although numerous cancer cells can find their way into the circulation, the majority will succumb during their journey, with only few able to extravasate, expand and successfully colonize other organs ${ }^{3}$. Clinical observations and multiple experi- mental studies have led to several propositions that can explain the acquired metastatic traits of cancer cells (as described and summarized elsewhere ${ }^{2-}$ ${ }^{7}$ ). Regardless, whether a sequential acquisition of random mutations in tumours may provide and select for metastatic traits in rare tumour clones ${ }^{8}$ or the metastatic predisposition of a tumour is already imprinted in the majority of cancer cells $s^{4-7}$, cancer cells need to be able to continuously adapt to changing environments during metastasis. Thus, only very few metastatic cancer cells with the appropriate adaptations will colonize at distant sites $^{2,9}$. This knowl- edge supports the proposition that cancers do not ran- domly metastasize but, rather, dependent on the tumour type, seed to specific organs. Around 1900, James Ewing argued that tumour cells seed in a circulationdependent manner ${ }^{10}$, while Stephen Paget proposed the 'seed and soil' hypothesis, stating that metastasizing cancer cells 'seed' only in certain especially hospitable tissues, akin to seeding in 'fertile soil ${ }^{11}$. Since then, subsequent studies have revealed several classes of metastasis genes whose altered activities are needed at individual steps along the metastatic process $^{9}$. The expression of gene

clusters associated with metastasis initiation and pro- gression has been shown to confer a selective advantage to cancer cells both at primary and secondary sites as well as in the circulation, whereas protein products of metastatic virulence genes only promote survival and proliferation during colonization at the metastatic site, suggesting also a specific role in their mediation of organ-specific metastase ${ }^{12-14}$. Organotropism is facili- tated by multiple factors including tumour-intrinsic fac- tors, organ-specific niches and the interaction between tumour cells and the host microenvironment ${ }^{15,16}$. Furthermore, through the secretion of specific factors and extracellular vesicles, tumours are able to establish a pre-metastatic niche in distant organs ${ }^{17,18}$. These fac- tors, for example, can activate distal lymphangiogenesis, recruit bone marrow-derived cells and promote extra- cellular matrix remodelling by activating stromal fibro- blasts, which creates a permissive microenvironment for cancer cell colonization ${ }^{19-21}$.

Only recently, attention has been drawn to the fact that metastatic cells also need to have or gain certain metabolic traits to be able to survive and grow in the new soil ${ }^{22-24}$, which may substantially vary in its nutri- ent and oxygen availability from the primary site. Such metabolic rewiring can be controlled transcriptionally, for example through epigenetic alterations, but also post-translationally or through metabolite availability to enzymes ${ }^{25}$. In this Review, we discuss growing evidence for the dynamic metabolic landscape metastasizing cells display to survive and propagate during the different steps of the metastatic cascade. 


\section{Metabolic adjustments during metastasis}

Accumulating evidence supports the presence of dynamic changes in the metabolism of metastasiz- ing cells, contributing to their ability to successfully transition through the changing microenvironments of the metastatic cascade. Here, we link the metabolic changes in cancer cells that traverse the metastatic cas- cade to the two intertwined concepts of metabolic plasticity and metabolic flexibility (FIG. 1). Similar to and extend- ing beyond the previously described divergence in metabolism ${ }^{24,26}$, metabolite plasticity describes metasta- sizing cells that can use one metabolite to fuel the various metabolic requirements of different steps of the meta- static cascade. In contrast, metabolite flexibility builds on the well-described nutrient flexibility of cells ${ }^{27,28}$ and refers to metastasizing cancer cells that can use different metabolites to meet the same metabolic requirement imposed by a specific step of the metastatic cascade.

\section{Metabolite plasticity}

It is conceivable that many nutrients can contribute to metabolite plasticity during metastasis formation. Yet the majority of metabolic research has focused on the early metastatic steps of tumour migration and invasion whereas only few nutrients have been analysed at sev- eral metastatic steps. Here, we discuss the implication of four such nutrients, namely lactate, pyruvate, glutamine and fatty acids, in regulating tumour invasion, survival in the circulation and colonization at a secondary site (FIGS 2,3; TABLE 1).

\section{Pyruvate and lactate metabolism}

Pyruvate is produced from glucose and other nutri- ents that fuel the glycolytic pathway, and can give rise to lactate in a one-step reaction catalysed by lactate dehydrogenase (LDH). In addition, pyruvate and, con- sequently, lactate can be produced from glutamine and other amino acids that fuel the tricarboxylic acid (TCA) cycle via malic enzyme (ME) or pyruvate carboxy kinase (PCK), which funnel TCA cycle-derived carbon towards the lower part of glycolysis. Pyruvate (and lactate) can be reversibly converted to alanine, resulting either in the production of alanine from pyruvate or vice versa. Additionally, pyruvate and, consequently, lactate can be oxidized in the mitochondria to acetyl-CoA by the enzyme pyruvate dehydrogenase (PDH) or converted by the enzyme pyruvate carboxylase (PC) to oxaloac- etate; the latter replenishes the TCA cycle (anaplerosis). Yet both lactate and pyruvate are also nutrients that can be taken up by cells directly from the environment.

Pyruvate and lactate metabolism in invading and motile cancer cells. One of the first metastatic attributes of cancer cells is their switch from a proliferative to a migrating phenotype. Multiple studies have provided evidence that metabolic changes in cancer cells mod- ulate activity of signalling pathways and global gene expression programmes driving migration and inva- sion (for example, an epithelial to mesenchymal transi- tion (EMT) ${ }^{23}$. These metabolic adaptations are not just consequential bystander effects because metabolites such as pyruvate and lactate can directly promote the invasion and migration ability of cancer cells (FIG. 2a). In breast cancer cells, the anaplerotic entry of pyru- vate via PC into the TCA cycle promoted an invasive phenotype by increasing their motility, although the underlying molecular mechanism remains elusive ${ }^{29,30}$. Pyruvate oxidation requires PDH activity. The result- ing acetyl-CoA production and further accumulation of acetyl-CoA, through phosphorylation-induced inhibi- tion of its consuming enzyme acetyl-CoA carboxylase 1 (ACC1) ${ }^{31}$, led to the acetylation of the transcription factor Smad2, which is a known inducer of mesenchy- mal gene expression patterns ${ }^{31}$. Gene expression analy- sis of 20 different human cancers has shown that poor survival was associated with inhibition of at least one mitochondrial pathway. Oxidative phosphorylation was the most affected pathway in patients with a low versus high survival rate, and most frequently coincided with downregulated gene expression of the genes encoding the subunits of Complex I and IV of the respiratory chain $^{32}$. Functionally, expression of genes contributing to oxidative phosphorylation showed a negative corre- lation with $\mathrm{EMT}^{32}$. Accordingly, metastasis-derived cell lines in vitro and metastases analysed ex vivo from a lung cancer mouse model had reduced mitochondrial functionality compared with non-metastatic primary tumours ${ }^{33}$. Although these changes in mitochondrial metabolism will have multiple effects, they may indi- cate a shift towards glycolytic energy production that requires lactate synthesis. Indeed, inhibition of lactate dehydrogenase A (LDHA) expression impaired invasion and migration in in vitro assays in renal cell carcinoma (RCC), pancreatic cancer and prostate cancer ${ }^{34-36}$ and decreased metastasis in an orthotopic renal xenograft model ${ }^{36}$. Moreover, downregulation of microRNA30a-5p suppressed LDHA expression and thereby inhibited gallbladder cancer progression as well as metastasis formation in breast cancer mouse models ${ }^{37,38}$, whereas knockdown of the oncogene c-MYC decreased LDHA expression in in vitro models of pancreatic cancer ${ }^{35}$. LDHA has also been found to be phosphorylated by HER2 and $\mathrm{SRC}^{39}$. Inhibition of this phosphorylation resulted in decreased invasiveness of head and neck as well as breast cancer cell lines in vitro and metastatic potential in breast cancer xenograft mouse model ${ }^{39}$. Interestingly, LDHA silencing could be rescued by lac- tate and the antioxidant $N$-acetylcysteine in in vitro assays, suggesting a redox metabolism-dependent mechanism ${ }^{39}$.

The transporters monocarboxylate transporter 1 (MCT1) and MCT4 preferentially exchange lactate between the extracellular and intracellular space ${ }^{40}$. Both proteins are independent prognostic markers for progression-free 
survival in clear cell RCC ${ }^{41}$, whereas high levels of MCT1 expression have been associated with a lower survival rate in patients with bladder cancer ${ }^{42}$. MCT1 expression also increased during glu- cose starvation in cervical cancer cell lines ${ }^{43}$ and during starvation-induced autophagy in hepatocellular carci- noma cell line $\mathrm{s}^{44}$, which are conditions that may occur in poorly perfused and hypoxic regions of the tumour microenvironment. Mechanistically, increased levels of MCT1 in cervical cancer cell lines can lead to the for- mation of a heterocomplex with CD147 (REF. ${ }^{43}$ ), a protein that stimulates secretion of matrix metalloproteinases (MMPs) and cytokines in many cancers ${ }^{45}$. This interac- tion was shown in oxidative phosphorylation-dependent tumour cells where it increased migratory capacities in vitro ${ }^{43}$. Notably, MCT1, independent of its lactate transport function, has been shown to activate NF- $\mathrm{B}$, which is an upstream regulator of a pro-invasive EMT in cervix squamous carcinoma cell lines and an experimen- tal mammary carcinoma mouse model ${ }^{46}$, and promoted migration and invasion in osteosarcoma cell lines ${ }^{47}$. Interestingly, $\mathrm{pH}$ changes, which may result from lactate production, also activated $\mathrm{NF}-\kappa \mathrm{B}$ in a breast cancer cell line ${ }^{48}$. In addition, lactate has been shown to promote breast cancer progression by supporting chemoattrac- tion, which stimulated cancer cell migration in a breast cancer cell line ${ }^{49}$. Accordingly, intraperitoneal admin- istration of lactate to mice increased lung metastasis formation resulting from intravenously injected breast cancer cells ${ }^{49}$. Recently, lactate has also been identified as a precursor of histone lactylation ${ }^{50}$. It will be very inter- esting to reveal whether this post-translational histone modification provides another regulatory mechanism in tumour invasion.

Taken together, the studies described above sup- port the notion that lactate and pyruvate metabolism can directly promote cancer invasion by inducing var- ious signalling pathways and molecules that drive and facilitate tumour cell migration and invasion.

Pyruvate and lactate metabolism in circulating tumour cells. Cancer cells need to increase their anti- oxidant defence in the circulation ${ }^{51-53}$ to avoid matrix detachment-induced cell death (anoikis, reviewed elsewhere ${ }^{54,55}$ ). Lactate and pyruvate metabolism may potentially contribute to resistance of matrix- detached cells against reactive oxygen species (ROS) (FIG. 2b). Studies in vitro and in patients revealed that pyruvate concentrations correlate with matrix detach- ment and invasiveness. As such, pyruvate levels were increased intracellularly in matrix-detached 293T cells ${ }^{56}$, whereas highly invasive ovarian cancer cells exhib- ited an increased pyruvate uptake in matrixdetached conditions compared with less invasive ovarian cancer cells ${ }^{57}$. In patients, levels of pyruvate were elevated in the plasma of individuals with metastatic carcinoma of different origin compared with healthy individuals ${ }^{58}$. Congruently, pyruvate concentrations in serum of patients with aggressive metastatic breast cancer were higher compared with those of patients with early-stage breast cancer ${ }^{59}$. Interestingly, a study in non-malignant and malignant cells indicates that pyruvate itself can act as an antioxidant via a non-enzymatic reac- tion with hydrogen peroxide ${ }^{60}$. Moreover, it has been demonstrated that the serum lactate concentration was higher in patients with metastatic compared with non- metastatic colorectal cancer ${ }^{61}$. Thus, these observations suggest that pyruvate and lactate are available to circulat- ing tumour cells and could support their successful tran- sition through the blood circulation. Corroborating the idea that pyruvate and lactate metabolism can protect against ROS, the disruption of intracellular conversion of pyruvate via PC to oxaloacetate in cultured breast cancer cells resulted in a decreased ratio of NADPH/NADP+ and glutathione (GSH)/GSSG, indicating reduced ROS scavenging capacity and, consequently, causing elevated oxidative stress ${ }^{62}$. Striking evidence has been provided for an important role of lactate metabolism in circulat- ing tumour cells. In particular, MCT1-mediated uptake of lactate facilitated melanoma metastasis in patient- derived xenografts by promoting pentose phosphate pathway flux and a consecutive ROS defence ${ }^{63}$. Matrixdetached cells can induce hypoxia via cell clustering to reduce $\mathrm{ROS}^{56}$. Interestingly, intracellular pyruvate or lactate accumulation can induce a hypoxic response by stabilizing hypoxia inducible factor $1 \alpha(\mathrm{HIF} 1 \alpha)^{64-66}$. In the case of lactate, this can also occur via $N$-Myc Downstream-Regulated Gene 3 Protein (NDRG3) stabilization ${ }^{67}$. Thus, it is tempting to specu- late that increased lactate and pyruvate concentrations in the blood circulation may support circulating tumour cells and cell clusters by facilitating a hypoxic response.

In conclusion, there is supporting evidence that lac- tate and pyruvate uptake can help circulating tumour cells to survive by enhancing their resistance against oxidative stress.

Pyruvate and lactate metabolism during metastatic col- onization. Once cancer cells reach a distant organ, they need to adapt to the new environment and also create a permissive niche in order to proliferate (FIG. 3; TABLE 1). Colonizing cancer cells are likely exposed to different types and/or levels of nutrients at the secondary site compared with the primary site. For example, pyru- vate is enriched in lung interstitial fluid compared with plasma ${ }^{68}$, with the lung being an organ of frequent meta- stasis across several cancer types. Thus, it is conceivable that cancer cells utilize pyruvate when undergoing meta- static colonization in the lung. Indeed, there is evidence from mouse models that breast cancer cells colonizing in the lung require extracellular pyruvate, but not lactate, to create a permissive niche through extracellular matrix remodelling ${ }^{69}$. In particular, increased levels of pyruvate facilitated transamination between glutamate and pyru- vate (catalysed by the mitochondrial enzyme alanine aminotransferase 2 (ALT2; also known as GPT2)), lead- ing to the generation of $\alpha$-ketoglutarate and alanine ${ }^{69}$. In turn, $\alpha$-ketoglutarate boosted activity of the enzyme col- lagen prolyl-4-hydroxylase (P4HA) ${ }^{69}$, which independent studies have reported to be essential for collagen depo- sition and remodelling ${ }^{70}$. Yet, the role of pyruvate in the lung environment may go beyond collagen hydroxylation because expression of PC was required for breast cancer- derived lung metastases but not for extrapulmonary metastases in an experimental mouse model $^{71}$. Moreover, pyruvate availability has been shown to 
increase activity of the serine biosynthesis pathway, which potentiated mTORC1 signalling in lung metastases but not primary breast tumours ${ }^{72}$.

Besides the above described findings, the number of studies that particularly investigate the impact of pyru- vate and lactate metabolism in metastatic colonization in an in vivo setting is rather limited. However, support- ing evidence for the importance of these two nutrients for metastatic colonization has been provided in vitro via colony formation assays, which may to some extent mirror a similar metabolic programme to that of in vivo metastatic colonization ${ }^{73}$. For example, pyruvate sup- plementation can stimulate in vitro colony formation in breast cancer cells by fuelling mitochondrial respiration ${ }^{74}$ and MCT1 inhibition with AR-C155858 had a cytostatic effect on colony growth in different cancer cell lines ${ }^{75}$. Similarly, blocking lactate and pyruvate entry into the mitochondria through inhibition of the mitochondrial pyruvate carrier (MPC) with 7ACC2 elicited cytotoxic effects on colony growth in different cell lines $^{75}$. Whereas there is evidence that pyruvate and lactate fuel in vivo tumour proliferation ${ }^{68,76}$, the full extent to which lactate and pyruvate are important nutrients for cancer cells to seed and colonize in the metastatic niche is largely unknown.

\section{Glutamine metabolism}

Glutamine is the most abundant free amino acid in the plasma. Many cancer cells take up glutamine, which contributes to non-essential amino acid as well as nucleo- tide synthesis through carbon or nitrogen metabolism. Moreover, glutamine can be converted in the mitochon- dria to replenish the TCA cycle (anaplerosis) or can be fully oxidized to produce ATP (glutaminolysis).

Glutamine metabolism in invading and motile cancer cells. Glutamine metabolism has been extensively stud- ied in proliferating cancer cells ${ }^{77}$. Emerging evidence suggest that glutamine metabolism is also important for invasion (FIG. 2a; TABLE 1). Invasive, but not non- invasive, ovarian cancer cells displayed a dependency on glutamine availability in vitro ${ }^{78}$, and metastatic mel- anoma cells exhibited elevated glutamine oxidation ${ }^{79}$. Moreover, mRNA expression levels of glutaminase 1 (GLS1), which catabolizes glutamine to glutamate, correlated with lymph node metastasis of colorectal cancer, and GLS1 expression was required for hypoxia- mediated cancer cell migration in vitro ${ }^{80}$. Moreover, SOX12 overexpression compared with control was found to promote colorectal cancer metastasis via GLS1 in experimental mouse models ${ }^{81}$. The isoenzyme GLS2 repressed hepatocellular carcinoma metastasis based on protein interactions with the small GTPase RAC1 $\left(\mathrm{REF}^{82}\right.$ ) and with Dicer ${ }^{83}$. The latter interaction inhib- ited the pro-invasive regulator and EMT transcription factor Snail ${ }^{83}$. Specifically, GLS2 binds to small GTPase RAC1 and inhibits its interaction with the RAC1 activa- tors guanine-nucleotide exchange factors, which in turn inhibits RAC1 to suppress cancer metastasis $^{82}$. Moreover, GLS2 also interacts with Dicer and stabilizes Dicer pro- tein to facilitate miR-34a maturation, and subsequently represses Snail expression ${ }^{83}$. Both interactions are independent of the catalytic function of GLS2.

Glutamine-derived glutamate can have different fates, including secretion via the cystine antiporter xCT (also known as SLC7A11), expression of which has been identified as a predictive marker of recurrence, tumour invasion, lymph node metastasis and venous invasion in patients with colorectal cancer ${ }^{84}$. Mechanistically, secre- tion of glutamate via xCT can lead to paracrine activ- ation of the metabotropic glutamate receptor GRM3, which in turn upregulates Rab27-dependent recycling of the transmembrane membrane type 1 (MT1)-MMP to promote the invasive behaviour of breast cancer cells ${ }^{85}$. Accordingly, xCT inhibition with sulfasalazine impaired lung metastasis through a ROSdependent p38 MAPK activation in breast cancer and oesophageal squamous cell carcinoma mouse models ${ }^{86,87}$. In the latter scenario, disruption of xCT enhanced homotypic cell- cell adhesion and attenuated cell-extracellular matrix adhesion $^{87}$.

Thus, cancer cells can rely on glutamine and cys- tine metabolism to alter cell adhesion and activate invasive cues.

Glutamine metabolism in circulating cancer cells. So far, evidence of glutamine metabolism in supporting circulating tumour cells is sparse (FIG. 2b,c). Reduced glutamine concentrations and markedly elevated gluta- mate concentrations have been identified in the plasma of patients with oesophageal squamous cell carcinoma presenting lymph node metastasis compared with those who did not present with metastasis ${ }^{88}$. In line with this, circulating tumour cells with tumour-initiating capacity (CD44//high versus CD44-/low) from patients with gastric cancer have increased expression of $\mathrm{xCT}^{89}$. Thus, it is conceivable that increased xCT expression may explain the above discussed elevated levels of glutamate in the plasma of patients with metastatic oesophageal squa- mous cell carcinoma because xCT overexpression may favour the secretion of glutamate. Yet, the intracellular metabolism of glutamate seems also to be linked to metastatic cancers. As such, glutamate dehydrogenase (GDH), which converts glutamate to $\alpha$ ketoglutarate, has been identified as a prognostic marker of colorec- tal cancer metastasis ${ }^{90}$. Indeed, GDH knockdown or inhibition with R162 attenuated anoikis resistance and decreased tumour metastasis through CamKK2-AMPK signalling in an LKB1-deficient lung cancer mouse model ${ }^{91}$. Thus, different fates of glutamine may affect circulating tumour cells. For a broader understanding and interpretation of the above described observations, further investigations are required. 
Recent studies have described changes in glutamine metabolism of colonizing cancer cells (FIG. 3; TABLE 1). In this respect, inhibition of the glutamine transporter ASCT2 (also known as SLC1A5) with short hairpin RNA knockdown was shown to impair primary pros- tate cancer growth and lung, but not liver, metastasis in a mouse model ${ }^{92}$. Accordingly, treatment of the VM-M3 mouse model of systemic metastatic cancer with the glu- tamine analogue 6diazo-5-oxo-l-norleucine (DON) reduced metastasis to the liver, lung and kidney ${ }^{93}$. Moreover, anti-xCT vaccination inhibited arising and established lung metastasis nodules in breast cancer mouse models ${ }^{94}$. On the molecular level, xCT activity regulated cancer stem cell self-renewal and the intra- cellular redox balance in breast cancer cells ${ }^{94}$. These data show that glutamine and cystine metabolism can support cancer cells to settle in distant organs.

\section{Fatty acid metabolism}

Fatty acids are an important fuel in anabolic and cata- bolic processes. Fatty acids can be synthesized de novo or taken up from the extracellular space. Newly syn- thesized fatty acids are often further desaturated to monounsaturated fatty acids, whereas the generation of most polyunsaturated fatty acids requires the uptake of essential fatty acids such as linoleic acid. Fatty acids are thereby important building blocks of lipids in cell membranes, and their desaturation status and double-bond position can define physical, chemical and biological properties such as membrane fluidity and peroxidation sensitivity ${ }^{95,96}$. Additionally, fatty acids can serve as important signalling molecules ${ }^{25,97}$. Moreover, many fatty acids can be oxidized in the mitochondria to acetyl-CoA, whereas specific long-chain and branched-chain fatty acids are oxidized in the peroxisomes.

Fatty acid metabolism in invading and motile cancer cells. Multiple studies have linked obesity to cancer progression, metastasis formation and mortality in several cancer types, including prostate cancer, melanoma and breast cancer ${ }^{98-101}$. Although several mechanisms can contribute to this correlation, lipids have been func- tionally implicated in several steps of the metastatic cascade (FIG. 2a; TABLE 1). Indeed, a metabolomics analysis of metastatic versus nonmetastatic oral squamous carcinoma cell lines showed differences in lipid metabolism ${ }^{102}$. In various cancers, increased fatty acid uptake, lipid accumulation and/or overexpression of genes encoding fatty acid transporters or other fatty acid metabolism genes induced invasive and migratory traits of cancer cells compared with control conditions, elevated the seeding capacity of tumour cells in distant organs in several mouse models ${ }^{103-106}$ and was associated with metastatic progression as well as poor prognosis in patients with various cancers ${ }^{105}$.

To explore a potential mechanistic link between fatty acid availability and metastasis formation, com- monalities in gene expression across fatty acid-rich and cancer-associated environments have been analysed. Thereby, CD36, a transmembrane protein that facilitates the import of fatty acids into the cell, was found to be induced in ovarian cancer cells when co-cultured with adipocytes ${ }^{107}$, in cervical cancer of mice in response to a fat-enriched compared with control diet ${ }^{108}$ and in vitro in oral squamous carcinoma cells exposed to palmitate (compared with nonpalmitate) supplementation in the culture media ${ }^{103}$. Congruently, the breast-associated adipocyte secretome enabled in vitro fatty acid uptake and invasiveness in breast cancer cells via induction of CD36 expression ${ }^{109}$. Moreover, CD36 was highly expressed in several metastasis-initiating compared with non-metastatic cancer cells ${ }^{103}$. Highlighting the relevance of these observations, elevated CD36 expression levels predicted poor prognosis in patients with clear cell $\mathrm{RCC}^{110}$ as well as glioblastoma ${ }^{111}$, and accelerated gastric cancer metastasis in experimental mouse models $\mathbf{s}^{112,113}$. Conversely, CD36 inhibition (with Nobiletin, sulfo- $N$-succinimidyl oleate or CD36-short hairpin RNA-mediated knockdown) impaired angio- genesis as well as migration and invasion of breast cancer cell lines ${ }^{114,115}$. Silencing of CD36 in preclinical mouse models of prostate cancer also reduced fatty acid uptake, as well as the abundance of oncogenic signal- ling lipids, and slowed cancer progression compared with control conditions ${ }^{116}$. Different mechanisms have been identified as potentially responsible for the ability of CD36 to facilitate cancer cell invasion and motility. CD36-associated fatty acid uptake also promoted an EMT in hepatocellular carcinoma cell lines ${ }^{106}$, whereas the pro-metastatic effect of CD36 in cervical cancer was synergistic with a TGF $\beta$-induced EMT in cell lines ${ }^{117}$.

Fatty acid binding proteins (FABPs) can likewise facilitate fatty acid uptake by cells. In this respect, expres- sion of FABP4 (also known as A-FABP) was associated with metastatic potential, cancer progression and mor- tality of patients with ovarian cancer, and expression of FABP5 (also known as E-FABP) was accompanied by these parameters in patients with clear cell RCC and colorectal cancer ${ }^{118-120}$ (FIG. 2b). FABP5 induced a pro-metastatic EMT phenotype in hepatocellular car- cinoma cell lines with FABP5 overexpression whereas knockdown cell lines showed the opposite phenotype ${ }^{121}$. Moreover, FABP5 silencing impaired MMP expres- sion in cervical cancer cells in vitro and in vivo ${ }^{122}$, and decreased invasion and migration of gastric cancer cell lines in vitro ${ }^{123}$. Additionally, FABP5 expression was required in conjunction with fatty acid synthase (FASN) and monoacylglycerol lipase (MAGL) to support prostate cancer progression in mouse models ${ }^{124}$. Similarly, overexpression of the liver-specific FABP1 (also known as LFABP) promoted angiogenic properties and migra- tion in hepatocellular carcinoma cell lines and increased the number of liver metastases ${ }^{125}$. Further, FABP1 is highly expressed in $44 \%$ of patients with melanoma, ena- bling melanoma cells to increase their invasive potential through uptake of adipocyte-derived lipids ${ }^{126}$. 
Cancer cells can also synthesize fatty acids de novo from various nutrients. In this respect, there is some evidence that de novo fatty acid synthesis contributes to the invasion and migration capacity of cancer cells. Inhibition of FASN with the compound orlistate impaired melanoma-induced metastases and angiogenesis in a mouse model ${ }^{127}$, and silencing of FASN attenuated CD44 expression-induced signalling and metastasis formation in colorectal cancer mouse models ${ }^{128}$. Mechanistically, FASN-mediated de novo lipogenesis regulated expres- sion of CD44 at a posttranscriptional level, possibly by altering its palmitoylation ${ }^{128}$. In an independent study, the proliferation, adhesion and migration of colorec- tal cancer cells in vitro was promoted by FASN-driven sphingolipid metabolism modulating focal adhesion signalling ${ }^{129}$.

Fatty acids, regardless of whether they are taken up or synthetized de novo, can be further processed in the cell. This includes monodesaturation via the isoenzymes stearoyl-CoA desaturase 1 (SCD1) and SCD5, both pro- ducing the same fatty acids, for example palmitoleate, or via the enzyme fatty acid desaturase 2 (FADS2). Although FADS2 is mainly known for its function in polyunsaturation, it was recently discovered as an alter- native metabolic route of monodesaturation in cancers leading to the production of the fatty acid sapienate ${ }^{130,131}$. SCD1 and SCD5 have been already studied in the con- text of cancer cell invasion and migration. High levels of SCD1 expression were prognostic in patients with colorectal cancer and supported cancer cell migration in vitro through monounsaturated fatty acid produc- tion compared with SCD1-silenced cancer cells ${ }^{132,133}$.

In line with this, blocking fatty acid desaturation with the SCD inhibitor CAY10566 and the FADS2 inhibi- tor SC26196 impaired NF-kB signalling, resulting in decreased stemness properties of ovarian cancer cells compared with vehicle-treated controls ${ }^{134}$. By contrast, blocking SCD1 with A939572 compared with vehicle treatment triggered endoplasmatic reticulum stress in several melanoma cells and ovarian cancer cells, which then led to enhanced invasion and metastatic dissemi- nation to the lung of mice ${ }^{135}$. Thus, SCD1 inhibition has led to controversial results, either reducing or increas- ing metastasis formation in melanoma (TABLE 1), which may be dependent on the presence of an endoplasmatic reticulum stress response and the Melanocyte Inducing Transcription Factor (MITF) status. Expression of SCD5 was suggested to reduce melanoma-derived metas- tasis by impairing the secretion of the extracellular matrix-modifying protein SPARC ${ }^{136}$. To date, it remains to be determined to what extent FADS2produced sapienate plays a role in metastasis formation.

Beyond desaturation, other fatty acid modifications can occur that foster cancer progression. For example, there is evidence that inhibition of cholesterol esteri- fication through targeting cholesterol acyltransferase (ACAT) suppressed the development and growth of metastatic lesions in prostate cancer ${ }^{137}$ as well as pan- creatic cancer ${ }^{138}$ mouse models and reduced in vitro cell migration in Lewis lung carcinoma cells ${ }^{139}$. In prostate cancer cell lines, inhibition of cholesterol esterification blocked secretion of Wnt3a through reduction of monounsaturated fatty acids, which limited Wnt3a acylation and, consequently, cancer cell migra- tion ${ }^{137}$, whereas in pancreatic cancer cell lines it induced endoplasmatic reticulum stress through cholesterol accumulation ${ }^{138}$.

Fatty acids can be oxidized to generate acetyl-CoA. Major fatty acid oxidizing organelles are the mito- chondria into which many long-chain fatty acids, such as palmitate, are transported depending on carnitine palmitoyltransferase 1 (CPT1). Elevated fatty acid oxidation has been observed in breast and ovarian cancer cells in co-culture with adipocytes compared with sole monocultures ${ }^{140,141}$, whereas CPT1A-silenced breast cancer cells lacked the ability to effectively drive tumour-associated lymphangiogenesis because of decreased VEGF-C and VEGF-D expression ${ }^{142}$. Further, a splice variant of $C P T 1 A$ supported cancer cell survival and invasiveness by promoting histone deacetylase (HDAC) activity in the nucleus through protein-protein interaction with HDAC1 (REF. ${ }^{143}$ ). In addition, fatty acid oxidation can generate mitochondrial ROS, which is known to facilitate an EMT in different cancer cell lines ${ }^{144}$. Finally, the breakdown of odd-chain fatty acids, cholesterol and certain amino acids can lead to the pro- duction of methylmalonic acid (MMA). This metabolite was recently found to be elevated in the serum of old compared with young human individuals and to drive cancer progression in breast cancer mouse models due to the induction of a Sox4-mediated EMT ${ }^{145}$.

In summary, fatty acid metabolism can increase tumour cell migration and invasion by altering cellular signalling cues and regulation of epigenetic modifiers.

Fatty acid metabolism in circulating cancer cells. Higher lipid serum concentrations have been detected in colorectal and breast cancer patients with distant metastases compared with patients without metastases ${ }^{146,147}$. There was, however, no correlation between lipid serum lev- els and metastases in patients with oral squamous cell carcinoma $^{148}$. Thus, it appears that, at least in certain tumour types, metastasizing cancer cells can be exposed to higher lipid levels in the blood circulation (FIG. 2b,c). Given that exogenous lipids could be metabolized by circulating cancer cells, this could suggest that their metabolism can potentially support survival in the cir- culation.

Congruently, blocking fatty acid oxidation by CPT1A silencing led to ROS accumulation in matrix- detached colorectal cancer cell lines ${ }^{149}$. The accumu- lation of ROS has been shown to be detrimental for matrix-detached cells in vitro ${ }^{150}$ and circulating tumour cells in vivo ${ }^{51,52,151}$. In line, fatty acid oxidation-generated acetyl-CoA supported calcium/calmodulin-dependent kinase II (CaMKII) activity in prostate cancer cells that resulted in reduced anoikis and cell migration ${ }^{152}$. Thereby, the presumed mechanism may depend on acetyl-CoA- derived CoA, generated locally 
by a yet to be identified acetyl-CoA hydrolysing reaction, and consecutive bind- ing to the calmodulin (CaM)-binding domain of CaMKII to promote CaM binding and activation of CaMKII at basal calcium concentrations ${ }^{153}$. Moreover, a recent study demonstrated in mice bearing patient-derived melanomas that cancer cells metastasizing through the lymphatic sys- tem conveyed reduced levels of ROS-induced ferroptosis compared with cells metastasizing through the blood vas- cular system ${ }^{154}$. This was based on increased availability of oleic acid-containing vesicles in the lymph compared with blood that allowed circulating cancer cells to take up oleate, resulting in less desaturated cell membranes and reduced sensitivity to lipid peroxidation ${ }^{154}$. Interestingly, cancer cells that disseminated through the lymph and then entered the blood circulation were protected from ferroptosis compared with cancer cells directly dissemi- nating into the blood circulation ${ }^{154}$. Thus, fatty acids may be available to and protective of certain circulating cancer cells, depending on the metastatic route.

\section{Fatty acid metabolism during metastatic colonization.}

Little is known about the availability of fatty acids in the different sites of metastasis relative to the primary tumour tissues but there is ample evidence that fatty acid uptake and metabolism can boost the nesting of metastasizing cancer cells in multiple organs (FIG. 3; TABLE 1). Whereas targeting CD36 had only minor to no effects on primary tumour growth, it dramatically impaired metastasis formation in the lungs and lymph nodes (and likely other organs) of mice bearing oral carcinomas ${ }^{103}$ (FIG. 3a,b). In line with this observation, long non-coding RNA LNMICC promoted lymph node metastasis through FABP5-mediated fatty acid metab- olism in cervical cancer mouse models ${ }^{155}$, whereas FABP4 inhibition increased $\alpha$-ketoglutarate concentra- tions and, consequently, increased DNA demethylation through regulation of ten-eleven translocase (TET) enzymes in vitro and reduced omental colonization in a mouse model of ovarian cancer ${ }^{156}$ (FIG. 3c).

In addition to fatty acid uptake, de novo fatty acid synthesis has also been implicated in the ability of can- cer cells to colonize a distant organ. Accordingly, FASN overexpression resulted in increased peritoneal metas- tasis of ovarian cancers in mice, and promoted cellu- lar colony formation and metastatic ability in vitro ${ }^{157}$ (FIG. 3c). CD147 knockout in hepatocellular carcinoma cells decreased fatty acid synthesis by impairing the Akt/mTOR signalling pathway and upregulated per- oxisome proliferator-activated receptor- $\alpha$ (PPAR $\alpha$ ), resulting in increased proliferation and metastasis for- mation compared with control in cell lines and a mouse model ${ }^{158}$. Similarly, hyperactivation of sterol regulatory element-binding protein (SREBP), a downstream tar- get of mTOR, by Pten and Pml double-null compared with Pten-null genetic modification of mouse prostates was capable of promoting prostate cancer metastasis by upregulating de novo fatty acid synthesis ${ }^{98}$ (FIG. 3b). In addition, the fatty acid monodesaturating enzyme SCD1 was induced in melanoma cells when they were co-cultured with lung fibroblasts, and genetically silenc- ing SCD1 in cancer cells impaired metastasis formation and prolonged their survival of mice injected with melanoma cells ${ }^{159}$. Yet it has also been shown that an increased ratio of monounsaturated to saturated fatty acids can result in mitochondrial dysfunction and, con- sequently, reduced breast cancer metastasis to the lung ${ }^{160}$ (FIG. 3a). In line with this and as discussed above, eleva- tion of saturated fatty acids, for example through SCD1 inhibition, increased melanoma-derived lung metastasis through inducing invasion in a mouse model ${ }^{135}$.

Little is known about the role of fatty acid oxidation in metastatic colonization. In a mouse model of ovarian cancer, salt-inducible kinase 2 (SIK2)-phosphorylated ACC and SIK2 overexpression compared with control promoted fatty acid oxidation required for effective metastasis formation ${ }^{141}$ (FIG. 3c). Moreover, inducible silencing of YAP in melanoma cells in mice showed that cancer cells were critically dependent on YAP-induced fatty acid oxidation to seed in the lymph node, but not in the $\operatorname{lung}^{161}$ (FIG. 3b).

In summary, fatty acid uptake, synthesis and modi- fication fosters the colonization of the metastatic niche through multiple mechanisms.

\section{Additional metabolic rewiring}

The studies described above infer several metabolic liabilities that can be potentially targeted while cancer cells transition through the metastatic cascade (TABLE 1). Notably, there are additional metabolic vulnerabilities that have been evaluated only for a limited number of metastatic steps, and thus were not possible to include as examples for metabolite plasticity. This includes, but is not limited to, acetate, serine, alanine, proline and aspar- agine metabolism, which are highlighted below (FIGS 2,3; TABLE 1). We do not address the metabolic rewiring of glucose metabolism during metastasis formation, which is covered in other recent reviews ${ }^{22,162,163}$.

Acetate metabolism. Acetate is a nutrient that can be converted to acetyl-CoA or produced from acetyl-CoA via different enzymes. Acetyl-CoA concentrations can be increased through downregulation of the enzyme acyl-CoA thioesterase 12 (ACOT12), which converts acetyl-CoA to acetate. Consequently, ACOT12 downreg- ulation by genetic silencing and subsequent acetyl-CoA accumulation in hepatocellular carcinoma cell lines facil- itated histone acetylation-induced activation of Twist2 (REF. $\left.{ }^{164}\right)$, a known inducer of EMT. Phenotypically, ele- vated acetyl-CoA concentrations led to increased inva- sive properties of hepatocellular carcinoma cells ${ }^{164}$, and similar observations 
have been made in breast cancer cells ${ }^{31,164}$ (FIG. 2a). By contrast, inhibition of the cytosolic enzyme acetyl-CoA synthase 2 (ACSS2; which converts acetate to acetyl-CoA), resulted in increased invasive- ness and migration of hepatocellular carcinoma cells due to a HIF2 $\alpha$-dependent induction of an EMT $^{165}$ (FIG. 2a). Acetate is available to tumours via the circulation and, potentially, through production by other organs such as the liver ${ }^{16,167}$. As such, through conversion to acetyl-CoA and contribution to the TCA cycle, acetate can serve as a bioenergetic fuel for breast cancer, non-small cell lung cancer, clear cell RCC, melanoma and endometrial cancer-derived brain metastases ${ }^{168}$ (FIG. $3 \mathrm{~d}$ ). Thus, further studies are needed to evaluate the role of acetate metab- olism and acetyl-CoA concentrations in metastasis formation.

\section{Serine, alanine, proline and asparagine metabolism.}

Serine, alanine, proline and asparagine are non- essential amino acids that can be produced by cells but are also available to differing extents in body fluids. Serine is produced from the glycolytic intermediate 3-phosphoglycerate, alanine is made from pyruvate, and proline and asparagine are often generated from glutamine. Serine, alanine and proline, but not aspara- gine, can be catabolized by human cells, resulting in pyru-vate production (serine, alanine) and TCA and/or urea cycle fuelling and/or glutamate/glutamine production (proline).

It has been reported that the expression levels of all serine biosynthesis enzymes were elevated in MDA-MB-231 breast cancer cells with enhanced bone metastatic abilities compared with the parental cell lines ${ }^{169}$. Expression levels of the first enzyme of the serine biosynthesis pathway, namely phosphoglycer- ate dehydrogenase (PHGDH), has been associated with lymph node metastasis in patients with non-small cell lung cancer or pancreatic cancer ${ }^{170,171}$ and high expres- sion levels of PHGDH have been linked to shorter overall survival of patients with breast cancer-derived liver metastasis ${ }^{172}$. Serine conversion to glycine (indi- cated by high levels of serine hydroxymethyltrans- ferase 2 (SHMT2) expression) has been prognostic in patients with breast cancer-derived lung metastasis ${ }^{172}$. In mice, PHGDH activity facilitated breast cancer- derived lung metastasis formation through main- taining mitochondrial redox homeostasis ${ }^{173}$ (FIG. 3a). Moreover, PHGDH inhibition by genetic silenc- ing or PH-755 treatment in mice impaired breast cancer-derived brain metastases due to low availabil- ity of serine in the brain ${ }^{174}$ (FIG. 3d) and it decreased mTORC1 signalling in lung metastases but not in primary breast cancers ${ }^{72}$ (FIG. 3a). These observations indicate a role of serine metabolism in metastasis formation.

The biosynthesis and catabolism of alanine depends on the reversible reaction catalysed by ALT1 (cytosolic) or ALT2 (mitochondrial), which convert alanine and $\alpha$-ketoglutarate to pyruvate and glutamate or vice versa. In a mouse model of primary breast cancer, inhibition of alanine catabolism by silencing ALT2 impaired pri- mary tumour growth, in particular, leading to elevated $\alpha$-ketoglutarate concentrations, resulting in HIF1 $\alpha$ degradation and subsequent reduced sonic hedgehog signalling ${ }^{175}$. Conversely, ALT2 silencing compared with control led to inhibition of alanine synthesis from pyru- vate in breast cancer-derived lung metastasis, thereby reducing the metastatic burden in an experimental mouse model, based on decreased $\alpha$-ketoglutarate concentrations impairing P4HA activity and, conse- quently, collagen hydroxylation ${ }^{69}$ (FIG. 3a). Interestingly, these data suggest that primary breast cancers rely on alanine catabolism, whereas breast cancer-derived lung metastases rely on alanine biosynthesis.

Asparagine and glutamine metabolism are inter- twined because limited glutamine availability makes asparagine an essential amino acid ${ }^{176}$. Interestingly, reducing the bioavailability of asparagine to cancer cells through knockdown of asparagine synthetase (ASNS), treatment with l-asparaginase or dietary asparagine restriction reduced lung metastasis without affecting the growth of the primary breast tumour in mice ${ }^{177}$. Mechanistically, ASNS silencing impaired the number of circulating tumour cells as well as invasiveness by pre- venting the induction of mesenchymal gene expression patterns ${ }^{177}$ (FIG. 2a).

Proline is synthesized and catabolized by different enzymes coupled to different cofactors, namely proline dehydrogenase (PRODH) and pyrroline-5-carboxylate reductase 1 (PYCR1) ${ }^{178}$. PYCR1 is highly expressed in patients with invasive ductal breast carcinoma ${ }^{179}$ and its levels have been predictive for lymph node metastasis in patients with non-small cell lung cancer ${ }^{180}$. Moreover, PYCR1 silencing reduced invasiveness in neuroblastoma cell lines with overexpression of MZF1-AS1 in vitro ${ }^{181}$, whereas proline starvation impaired clonicity in differ- ent cancer cell lines ${ }^{182}$. Accordingly, the proline cycle consisting of catabolism via PRODH and synthesis via PYCR1 is required for breast cancer-derived lung metastasis in mice $^{73}$ (FIG. 3a).

Taken together, nutrients such as lactate, pyruvate, glutamine and lipids (and likely others) appear to be crucial metabolites in many steps of the metastatic cascade - that is, their use is plastic. Although the evi- dence presented here supports the concept of metab- olite plasticity, several questions arise. For example, it remains elusive as to how the cancer cell origin impacts the different aspects of metabolite plasticity-driven metastasis formation. The largest body of evidence for metabolite plasticity is based on a wide range of differ- ent in vitro and in vivo models across multiple cancer types. Thus, additional studies are required that follow metabolite plasticity within the same cancer model to link changes in the metabolization of a particular nutri- ent to phenotypic switches of the cancer cells. Moreover, it will be important to understand how concentration changes of one and the same metabolite can arise 
across different organelles, tissues, tumours and patients and how these contribute to the ability of cancer cells to leverage these metabolites during metastasis forma- tion. Interestingly, some of the targets discussed above also affect primary tumours, whereas others only affect metastasis formation (BOX 1; TABLE 1). The latter supports the proposition that the metastatic phenotype and/or the site of metastasis defines the metabolic vulnerabilities of cancer cells.

\section{Metabolite (in)flexibility}

Multiple studies demonstrate that cancer cells rely on different nutrients to fuel the same metabolic require- ments, a phenomenon termed metabolite flexibility, which might allow them to overcome the hurdle of a specific step in the metastatic cascade. Here, we will discuss metabolite flexibility in light of circulating and colonizing tumour cells. We decided to cover these two specific steps in the metastatic cascade because there is evidence that circulating cancer cells require certain metabolic products (that is, NADPH, glutathione) to control ROS levels, whereas cancer cells that seed and colonize the metastatic niche have an increased need for the metabolic product ATP compared with prolifer- ating cancer cells in the primary tumour ${ }^{23,24,183,184}$. ATP and NADPH can be produced from various nutrients; however, ATP and NADPH in metastasizing cancer cells seem to be produced from only a limited range of nutrients (nutrient inflexibility).

\section{ROS defense in circulating tumour cells}

Antioxidant metabolism and resistance to ROS is essen- tial for the survival of cancer cells in the circulation ${ }^{23,24}$. Cancer cells can rely on different nutrients to avoid ROS-induced cell deaths such as ferroptosis (FIG. 4). As described above, pyruvate can act as an extracellular antioxidant ${ }^{60}$ whereas lactate-driven pentose phosphate pathway activity ${ }^{63}$ and fatty acid oxidation ${ }^{185}$ generate NADPH. NADPH is required for the recovery of the ROS scavenger GSH in melanoma and colorectal cancer, and potentially other cancers. As mentioned above, oleic acid uptake reduced the susceptibility of lymph-circulating melanoma cells to ferroptosis in blood circulation through the reduction of peroxidation-sensitive dou- ble bounds in lipids ${ }^{154}$. In addition, glutamine reductive carboxylation and glucose fermentation induced through cell detachment protected cells from anoikis by NADPH regeneration in the mitochondria and reduced mitochon- drial ROS production in various cancer cell lines ${ }^{150,186}$. Moreover, glucose-fuelled folate metabolism reduced oxidative stress in circulating melanoma cells based on low-dose methotrexate treatment, ALDH1L2 knockdown or MTHFD1 knockdown inhibiting distant mel- anoma metastasis but not primary tumours in mouse ${ }^{51}$. These findings highlight that numerous nutrients, including pyruvate, lactate, fatty acids, glutamine and glucose, can contribute to ROS resistance in circulating tumour cells.

\section{ATP production in seeding tumour cells}

Energy generation has emerged as an important meta- bolic output for cancer cells establishing tumours in distant organs $^{23}$. The reason for increased energy requirement of cancer cells colonizing in distant organs is unknown; however, it is conceivable that this could relate to protein as well as extracellular matrix production and trafficking, which are both highly energy-demanding processes ${ }^{187}$ and required to form a permissive metastatic niche. Cancer cells can rely on different nutrients to increase their ATP availability during metastatic coloni- zation (FIG. 4). Fatty acids have been identified as impor- tant nutrients in this respect. In breast cancer, metastatic triple-negative breast cancer cells compared with benign cell lines required fatty acid-driven energy metabolism for the formation of distant metastasis based on the inhi- bition of fatty acid oxidation with etomoxir or the per- turbance of fatty acid metabolism through inhibition of CUB-domain containing protein 1 (CDCP1) activity with an engineered blocking fragment in experimen- tal mouse models ${ }^{188,189}$. The rewiring of energy metab- olism yielded more ATP that was needed to activate Src through autophosphorylation ${ }^{188}$. Moreover, both proline catabolism and glucose metabolismderived ATP con- tributed to energy production during metastatic colo- nization of breast cancer cells in the lung of mice $^{68,190}$. Interestingly, breast cancer-derived lung metastases used glucose to fuel glycolysis and mitochondrial metabolism to account for their energy needs ${ }^{190}$, whereas breast cancer-derived liver metastases only relied on glycolytic energy production ${ }^{191}$. In mouse models of colon cancer, colon cancer-derived liver metastases scavenged ATP from the extracellular space ${ }^{192}$. In particular, colon cancer cells released creatine kinase brain-type into the liver extracellular space, which converted creatine into phosphocreatine in an ATP-driven reaction ${ }^{192,193}$. Phosphocreatine was then taken up by the colonizing colon cancer cells and used for intracellular phosphoryl- ation of ADP to ATP. In summary, there is evidence that cancer cells, in principle, can rely on several metabolites (for example, fatty acids, proline, glucose) to accom- modate their energy needs when they colonize distant organs.

The fact that cancer cells rely on multiple metabolites and nutrients to produce NADPH, GSH and ATP is not surprising, especially as these metabolic products are substrates and products of multiple reactions across the entire metabolic network. It is, however, surprising that targeting only one of the nutrients producing these molecules is sufficient to exhibit therapeutic efficacy in mouse models; for example, leading to reduction of the number of circulating tumour cells or reduced metas- tasis formation (FIG. 4). Thus, metastasizing cancer cells entail a certain metabolic rigidity, that is, nutrient inflexibility that seems to depend on the organ of metas- tasis, cell state, cell of 
origin, microenvironment or (epi) genetic landscape, which opens a therapeutic window to target these metabolic vulnerabilities. Accordingly, proline catabolism inhibition through blocking PRODH or inhibition of lactate uptake through MCT1 did not impair primary tumour growth, whereas it had dramatic effects on metastasis formation due to the different cell state and/or microenvironment during metastasis ${ }^{63,73}$.

Consequently, interesting questions arise such as which patients would benefit most from such treat- ments; and whether an anti-metabolic therapy would be more effective and eventually less toxic than a stand-ard chemopreventative strategy in counteracting the metastatic seeding, or would also be considered for patients who already have developed metastatic dis- ease. If organ-specific metastases may indeed demon- strate specific metabolic vulnerabilities that are targetable, anti-metabolic therapy of patients with established metastases might become an achievable goal. Considering the possibility that some of the vulnera- bilities of metastasizing cancer cells, such as increased energy needs during seeding or sensitivity of circulating tumour cells to oxidative stress, may be lost in estab- lished metastases further argues for distinct therapeutic windows in which metabolic targeting therapies would be most effective. Clinical trials in the neoadjuvant set- ting may thus be a promising strategy to evaluate meta- bolic therapeutics given that tumour spread has become an accepted clinical trial end point ${ }^{194}$.

\section{Metabolic evolution of metastases}

Once cancer cells have successfully metastasized to a distant organ, the secondary tumour shows a similar behaviour (that is, phenotype) to the primary tumour - both grow, proliferate and can reseed ${ }^{2,9}$. One could then argue that primary tumours and established metastases are metabolically similar, or at least depend on the same metabolic pathways or enzymes. Some examples dis- cussed above are in line with this proposition because, in some cases, disrupting metabolic activity leads to effects in cancer cells regardless of whether they grow as metas- tases or primary tumours (TABLE 1). However, there is an increasing body of evidence that suggests a metabolic evolution from primary tumours to metastases in differ- ent organs. Accordingly, some metabolic vulnerabilities discussed above only target metastases (sometimes only in specific organs) but not primary tumours (TABLE 1). Here, we summarize the current evidence suggesting that primary cancers and their metastatic progenies differ in their metabolic profiles.

Over the last decade, expression analysis of a lim- ited number of metabolic genes has revealed distinct expression profiles between primary and secondary lesions. For example, a study on patients with pancreatic adenocarcinoma and corresponding metastatic lesions demonstrated common but also highly distinct meta- bolic gene expression profiles between primary tumours and metastases ${ }^{195}$. A recent single-cell RNA-sequencing study of metastatic patient-derived xenograft tumours confirmed a clear intra-tumour and inter-tumour heterogeneity in metabolic expression profiles in distinct cancer cell populations in primary breast tumours, and lung and lymph node micrometastases ${ }^{196}$. Additionally, single-cell transcriptional profiling of tumour tissue samples from six patients with triple-negative breast cancer showed subclonal heterogeneity of malignant cells shared by various tumours with multiple signatures of treatment resistance and metastasis as characterized by elevation of glycosphingolipid metabolism and asso- ciated innate immunity pathways ${ }^{197}$. Moreover, lung micrometastases from breast cancer patient-derived xenograft mouse models displayed a distinct metabolic gene expression signature of mitochondrial oxidative metabolism compared with the corresponding primary breast cancers ${ }^{196}$. These results support the notion that metastases can be metabolically different compared with their corresponding primary tumours. Notably, whereas the evidence presented here and below is mainly based on transcriptional changes, post-translational modifi- cations and metabolite concentration-driven metabolic rewiring in primary tumours versus metastasis is likely to occur, yet is less demonstrated due to technological limitations.

Another layer of complexity is observed by inter- metastatic metabolic heterogeneity in relation to their metastatic site. Data from Flura-seq (fluorouracil- labelled RNA sequencing), a technique that determines which genes are active in small clusters of cells in a tis- sue, showed that lung micrometastases had differential transcriptional activity compared with brain microme- tastases and primary mammary tumours in mice ${ }^{198}$. This may suggest that metabolic requirements exist in the lung microenvironment that are distinct from brain or breast tissue. Accordingly, mitochondrial electron transport chain genes were higher expressed in lung metastases compared with both brain metastases and orthotopic mammary tumours ${ }^{198}$. In addition, a pro- teomics study on patient-derived breast cancer cells observed a unique metabolic protein profile of brain metastasis compared with bone metastasis in mice, which suggests either a selection of predisposed cells or bioenergetic adaptation of the tumour cells to the brain environment ${ }^{199}$. In line with this, melanoma-derived brain metastases displayed an enrichment for oxida- tive phosphorylation-associated gene expression pat- terns in comparison with patient-matched extracranial metastases $^{200}$.

Although these observations reiterate the existence of differential metabolic traits between primary tumours and metastases, and across metastases, the need for these metabolic alterations are less understood. At least two fundamental principles could lay the foundation for these alterations (FIG. 5). Either the (epi)genetically or metabolically heterogeneous tumour cell pool in a pri- mary tumour provides a selection for a distinct cancer cell subpopulation that is optimally suited to flourish in a specific organ environment, or several cancer cell 
subpopulations may be able to adapt to a certain organ environment. It is conceivable that both selective and adaptive processes occur, which may again depend on the tumour origin and metastatic site.

Regardless of whether selection or adaptation is the determinant factor for successful growth of secondary tumours, emerging evidence shows that the (metabolic) environment matters. An interesting experiment com- pared the influence of in vitro and in vivo environments. Thereby, brain and lung metastases as well as mammary tumours from mice were directly analysed after harvest- ing the tissue ${ }^{198}$. In parallel, an aliquot of these tissue samples was dissociated into single cells, cultured for 1-2 weeks and subjected to RNA sequencing. Strikingly, several thousand genes were distinctly expressed across the different tissues whereas the same cells showed dif- ferential expression of only a few hundred genes when cultured in vitro ${ }^{198}$. Moreover, in vitro culture of lung cancer cells versus in vivo growth of the same tumour cells resulted in a different metabolic phenotype ${ }^{201}$. Moreover, microenvironmental differences in per- fusion also correlated with intra-tumour metabolic differences in patients with lung cancer ${ }^{202}$. Such exper- iments demonstrate the environmental dependency of metabolism.

In addition, there is also circumstantial evidence that the nutrient availability in the environment is important. For example, breast cancer-derived brain metastases, similar to glioblastoma, can use acetate for propagation ${ }^{168,203}$ (FIG. 3d). This observation was based on ${ }^{13} \mathrm{C}$ tracer infusions in humans and mice, which assess in vivo nutrient contribution to metabolism ${ }^{204,205}$. Moreover, there is evidence that secreted factors from primary breast tumours increased the glucose availabil- ity in the metastatic niche, which elevated the effective- ness of metastatic seeding in the lungs of mice ${ }^{206}$ (FIG. 3a). Thus, nutrient availability in the metastatic niche is important and can be altered to increase the permis- siveness of the niche. Data from ${ }^{13} \mathrm{C}$-glucose infusions in mice harbouring metastatic primary breast cancer demonstrated an increase in PC-dependent anaplero- sis in lung metastases compared with primary tumours, which was recapitulated in vitro by adding pyruvate to the media ${ }^{68}$. Additionally, the dependence of breast cancer-derived brain metastases on PHGDH activity can be explained through the very low serine availability in the brain environment ${ }^{174}$ (FIG. 3d). Finally, there is also some evidence that the microenvironment may prepare cancer cells en route metabolically for another environ- ment because melanoma cells that traversed through the oleate-enriched environment of the lymph system before entering the blood circulation were better prepared to avoid cell death, and consequently more successful in seeding in distant organs of mice ${ }^{154}$ (FIG. 2b).

The studies described above support the notion that primary tumours and their metastases differ in their metabolic attributes because metastases adapt or have growth advantages for specific environments (FIG. 5). Although limited evidence exists, metabolic priming of the metastatic niche ${ }^{206}$ and by a certain environment ${ }^{154}$ may also exist. Whereas the here presented evi- dence suggests a role of the nutrient environment, additional factors of the microenvironment such as the presence of stromal cells may also contribute to environment-dependent metabolic requiring. Thus, it is tempting to speculate that metastases of differing origin growing in the same organ or taking the same route of metastasis may metabolically be more similar to each other than metastases of the same origin growing in different organs.

It is important to note that the environment is cer- tainly not the only determinant of metabolic rewiring and that other factors such as the cancer cell origin, that is the cell lineage from which the cancer cell arises, may override the influence of nutrient availability (as sug- gested for PCG1 $\alpha$ in breast versus prostate cancer ${ }^{23}$ ). Moreover, some metastatic niches may be more permis- sive for cancer cells with different genetically embed- ded metabolic programmes to successfully proliferate. Finally, some metabolic traits such as the reliance on CD36 activity in metastasis-initiating cells seem to be essential across multiple cancers and metastatic sites ${ }^{103}$, making them very attractive targets for cancer therapy.

Further studies that dissect the metabolic dependen- cies of metastases will be instrumental in determining and mechanistically understanding when the influence of the microenvironment outweighs the cellular origin, cell state (that is, phenotype) and (epi)genetic landscape in imposing metabolic constrains on metastasis growing in different organs.

\section{Conclusion}

Emerging evidence depicts specific metabolic vulnera- bilities in cancer cells during their metastatic journey that could be exploited to potentially halt metastatic growth and even prevent successful seeding. These vulnerabilities are not just manifested intrinsically in a tumour type-specific manner but, rather, dynamically dependent on the stage of and location during their metastatic journey. Importantly, the success of metastatic manifestation may be influenced by the nutrient compo- sition of the respective organ to which metastatic tumour cells need to adapt. These observations fit well with the 'seed and soil' hypothesis in which tumour cells require an appropriate soil with the necessary metabolites and nutrients in order to expand successfully. Nonetheless, the complexity of metabolic traits and their necessities in maintenance and propagation, not only of tumour cells but of every normal cell, makes therapeutic targeting of metabolic pathways challenging and requires a more detailed understanding of the effects of metabolite inhi- bition in the context of the heterogeneous cell types in metastases and the organ in which they seed. 
1. Fares, J., Fares, M. Y., Khachfe, H. H., Salhab, H. A. $\&$ Fares, Y. Molecular princi hallmark of cancer revisited. Sig. Transduct Target Ther. 5, 28 (2020).

2. Lambert, A. W., Pattabiraman, D. R. \& Weinberg, R. A. Emerging biological principles of metastasis. Cell 168 , 670-691 (2017).

3. Vanharanta, S. \& Massague, J. Origins of metastatic traits. Cancer Cell 24, 410-421 (2013).

4. Steeg, P. S. Targeting metastasis. Nat. Rev. Cancer 16 201-218 (2016).
5. Turajlic, S. \& Swanton, C. Metastasis as an evolutionary process. Science 352, 169-175

6. Plaks, V., Kong, N. \& Werb, Z. The cancer stem cell niche: how essential is the niche in regulating stemness of tumor cells? Cell Stem Cell 16, 225-238 (2015)

7. Gonzalez, H., Hagerling, C. $\&$ Werb, Z. Roles of the immune system in cancer: from tumor initiation to metastatic progression. Genes Dev. 32, 1267-1284 (2018)
8. Fidler, I. J. \& Kripke, M. L. Metastasis results from preexisting variant cells within a malignant tumor. Science 197, 893 (1977).

9. Valastyan, S. \& Weinberg, R. A. Tumor metastasis: molecular insights and evolving paradigms. Cell 147 275-292 (2011)

10. Ewing, J. Neoplastic Diseases: A Treatise on Tumors (W. B. Saunders, 1928).

11. Paget, S. The distribution of secondary growths in cancer of the breast. Lancet 133, 571-573 (1889). 
12. Nguyen, D. X., Bos, P. D. \& Massagué, J. Metastasis: from dissemination to organ-specific colonization. Nat. Rev. Cancer 9, 274-284 (2009).

13. Pavlovic M et al. Enhanced MAF oncogene expression and breast cancer bone metastasis. J. Nat Cancer Inst. 107, djv256 (2015)

14. Ganesh, K. et al. L1CAM defines the regenerative origin of metastasis-initiating cells in colorectal cancer. Nat. Cancer 1, 28-45 (2020).

15. Gao, Y. et al. Metastasis organotropism: redefining the congenial soil. Dev. Cell 49, 375-391 (2019).

16. Tian, X. et al. Organ-specific metastases obtained by culturing colorectal cancer cells on tissue-specific decellularized scaffolds. Nat. Biomed. Eng. 2,

Peinado, H. et al. Pre-metastatic niches: organ-specific for metastases. Nat. Rev. Cancer 17, 302-317 (2017).

Doglioni, G., Parik, S. \& Fendt, S.-M. Interactions in the (pre)metastatic niche support metastasis

19. Kaplan, R. N et al VEGFR1-positive haematopoietic bone marrow progenitors initiate the pre-metastatic bone marrow progenitors initiate the
niche. Nature 438, 820-827 (2005)

20. Hoshino, A. et al. Tumour exosome integrins determine organotropic metastasis. Nature $\mathbf{5 2 7}$ 329-335 (2015).

21. Olmeda, D. et al. Whole-body imaging of lymphovascular niches identifies pre-metastatic roles of midkine. Nature 546, 676-680 (2017).

22. Teoh Shao, T. \& Lunt Sophia, Y. Metabolism in cancer metastasis: bioenergetics, biosynthesis, and beyond. Wiley Interdiscip. Rev. Syst. Biol. Med. 10, e 1406 (2018).

23. Elia, I., Doglioni, G. \& Fendt, S.-M. Metabolic hallmarks of metastasis formation. Trends Cell Biol. 28, 673-687 (2018).

24. Faubert, B., Solmonson, A. \& DeBerardinis, R. J. Metabolic reprogramming and cancer progression. Science 368, eaaw5473 (2020)

25. Lorendeau, D., Christen, S., Rinaldi, G. \& Fendt, S.-M. Metabolic control of signaling pathways and metabolic auto-regulation. Biol. Cell 107, 251-272 (2015).

26. Kim, J. \& DeBerardinis, R. J. Mechanisms and implications of metabolic heterogeneity in cance Cell Metab. 30, 434-446 (2019).

27. Kelley, D. E. $\&$ Mandarino, L. J. Fuel selection in human skeletal muscle in insulin resistance:

a reexamination. Diabetes 49,677-683
Randle, P. J., Garland, P. B., Hales, C. N. \&

Newsholme, E. A. The glucose fatty-acid cycle. Its role in insulin sensitivity and the metabolic disturbances of diabetes mellitus. Lancet 1, 785-789 (1963).

29. Phannasil, $P$. et al. Mass spectrometry analysis shows the biosynthetic pathways supported by pyruvate carboxylase in highly invasive breast cancer cells. 537-551 (2017).

30. Phannasil, P. et al. Pyruvate carboxylase is up-regulated in breast cancer and essential to support growth and invasion of MDA-M

31. Rios Garcia, M. et al. Acetyl-CoA carboxylase 1-dependent protein acetylation controls breast cancer metastasis and recurrence. Cell Metab. 26, 842-855.e5 (2017)

32. Gaude, E. \& Frezza, C. Tissue-specific and convergent metabolic transformation of cancer correlates with metastatic potential and patient survival. Nat. Commun. 7, 13041 (2016)

33. Chuang, $\mathrm{C}-\mathrm{H}$. et al. Altered mitochondria functionality defines a metastatic cell state in lung cancer and creates an exploitable vulnerability. Cancer Res. https://doi.org/10.1158/0008-5472.CAN-20-1865 (2020)

34. Xian, Z.-Y. et al. Inhibition of LDHA suppresses tumor progression in prostate cancer. Tumor Biol. 36 8093-8100 (2015)

35. He, T.-L. et al. The c-Myc-LDHA axis positively regulates aerobic glycolysis and promotes tumor progression in
187 (2015).

36. Zhao, J. et al. LDHA promotes tumor metastasis by facilitating epithelial-mese cell carcinoma. Mol. Med. Rep. 16, 8335-8344 (2017).

37. Li, L. et al. miR-30a-5p suppresses breast tumor growth and metastasis through inhibition of LDHAmediated Warburg effect. Cancer Lett. 400, 89-98 (2017)

38. He, Y. et al. LDHA is a direct target of miR-30d-5p and contributes to aggressive progression of gallbladder
carcinoma. Mol. Carcinog. 57, 772-783 (2018).
39. Jin, L. et al. Phosphorylation-mediated activation of LDHA promotes cancer cell invasion and tumour metastasis. Oncogene 36, 3797 (2017).

40. Pérez-Escuredo J et al. Monocarboxylate transporters in the brain and in cancer. Biochim Biophys. Acta 1863, 2481-2497 (2016).

41. Cao, Y.W. et al. Monocarboxylate transporters MCT and MCT4 are independent prognostic biomarkers for the survival of patients with clear cell renal cell carcinoma and those receiving therapy targeting angiogenesis. Urol. Oncol. 36, 311.e315-311.e25 (2018)

42. Zhang, G. et al. MCT1 regulates aggressive and metabolic phenotypes in bladder cancer. J. Cancer 9 2492-2501 (2018)

43. De Saedeleer, C. J. et al. Glucose deprivation increases (MCT1) expression and MCT1-dependent tumor cell migration. Oncogen 33, 4060 (2013)

44. Fan, Q. et al. Autophagy promotes metastasis and glycolysis by upregulating MCT 1 expression and Wnt/ $\beta$-catenin signaling pathway activation in hepatocellular carcinoma cells. J. Exp. Clin. Cancer Res. 37, 9 (2018)

45. Xiong, L., Edwards, C. K. 3rd \& Zhou, L. The biological function and clinical utilization of CD147 in human diseases: a review of the current scientific literature Int. J. Mol. Sci. 15, 17411-17441 (2014).

46. Payen V. L et al Monocarboxylate transporter MCT promotes tumor metastasis independently of its activity as a lactate transporter. Cancer Res. 77,5591 (2017)

47. Zhao, Z. et al. Downregulation of MCT1 inhibits tumor growth, metastasis and enhances chemotherapeutic NF-kB pathway Cancer Lett 342,150-158 (2014).

8. Gupta, S. C. Singh R. Pochampally R W (2014). $\&$ Mo, Y.Y. Acidosis promotes invasiveness of breast cancer cells through ROS-AKT-NF-kB pathway. Oncotarget 5, 12070-12082 (2014).

49. Bonuccelli, G. et al. Ketones and lactate "fuel" tumor growth and metastasis. Cell Cycle 9, 3506-3514 (2010)

50. Zhang, D. et al. Metabolic regulation of gene expression by histone lactylation. Nature $\mathbf{5 7 4}$ 575-580 (2019).

51. Piskounova, E. et al. Oxidative stress inhibits distant metastasis by human melanoma cells. Nature 12 186-191 (2015)

This article provides evidence that antioxidants increase the survival of melanoma cells in the circulation.

52. Le Gal, K. et al. Antioxidants can increase melanoma metastasis in mice. Sci. Trans/ Med. 7, 308re308 (2015)

This article provides evidence that antioxidants and the glutathione synthesis can increase lymph node metastasis formation of melanoma.

53. Wiel, C. et al. BACH1 stabilization by antioxidants stimulates lung cancer metastasis. Cell $1 \mathbf{7 8}$ 330-345.e22 (2019).

54. Guadamillas, M. C., Cerezo, A. \& del Pozo, M. A. Overcoming anoikis - pathways to anchorageindependent growth in cancer. J. Cell Sci. 124, 3189 (2011)

55. Grossmann, J. Molecular mechanisms of "detachmentinduced apoptosis - anoikis". Apoptosis 7, 247-260 (2002)

56. Labuschagne, C. F., Cheung, E. C., Blagih, J., Domart, M.-C. \& Vousden, K. H. Cell clustering promotes a metabolic switch that supports metastatic colonization. Cell Metab. 30 720-734.e725 (2019)

This work reveals hypoxia-induced mitophagy of clustering tumour cells in the circulation that limits mitochondrial ROS production, and promotes survival and metastasis formation.

57. Caneba, C. A., Bellance, N., Yang, L., Pabst, L. \& Nagrath, D. Pyruvate uptake is increased in highly nvasive ovarian cancer cells under anoikis conditions for anaplerosis, mitochondrial function, and migration. Am. J. Physiol. Endocrinol. Metab. 303 , E1036-E1052 (2012)

58. Maneche, H. C. Blood pyruvate in malignant eoplastic disorders. Clin. Chem. 12, 158-164 (1966).

59. Jobard, E. et al. A serum nuclear magnetic resonance based metabolomic signature of advanced metastatic human breast cancer. Cancer Lett. 343, 33-41 (2014)

60. Donnell-Tormey, J., Nathan, C. F., Lanks, K. DeBoer, C. J. \& de la Harpe, J. Secretion of pyruvate.
An antioxidant defense of mammalian cells. J. Exp. Med. 165, 500 (1987).

61. Wei $Y$ et al Prognostic significance of serum lactic acid, lactate dehydrogenase, and albumin levels in pestients with metastatic colorectal cancer, BioMed. Res. Int 2018, 1804086 (2018).

62. Wilmanski, T. et al. Inhibition of pyruvate carboxylase by 1a,25-dihydroxyvitamin $D$ promotes oxidative stress in early breast cancer progression. Cancer Lett. 411, 171-181 (2017)

63. Tasdogan, A. et al. Metabolic heterogeneity confers differences in melanoma metastatic potential. Nature

This study shows that MCT 1-dependent lactate uptas circulation.

64. Vande Voorde, J. et al. Improving the metabolic fidelits of cancer models with a physiological cell culture medium. Sci. Adv. 5, eaau7314 (2019).

65. Zera, K. \& Zastre, J. Stabilization of the hypoxiainducible transcription factor-1a (HIF-1 $\alpha$ ) in thiamine deficiency is mediated by pyruvate accumulation. Toxicol Appl Pharmacol 355, 180-188 (2018).

6. Saedeleer C. J et al. Lactate activates HIF-1 in oxidative but not in Warburg-phenotype human tumor cells. PloS ONE 7, e46571 (2012).

7. Lee, D. C. et al. A lactate-induced response to hypoxia Cell 161, 595-609 (2015)

68. Christen, S. et al. Breast cancer-derived lung metastasis show increased pyruvate carboxylase (2016).

69. Elia, I. et al. Breast cancer cells rely on environmental pyruvate to shape the metastatic niche. Nature $\mathbf{5 6 8}$, 117-121 (2019).

This article demonstrates that breast cancer cells are dependent on pyruvate uptake to initiate collagen-based extracellular matrix remodelling for the establishment of a metastatic niche and subsequent metastatic growth in the lung.

70. Gilkes, D. M. et al. Collagen prolyl hydroxylases are essential for breast cancer metastasis. Cancer Res. 73, 3285-3296 (2013).

71. Shinde, A., Wilmanski, T., Chen, H., Teegarden, D. $\&$ Wendt, M. K. Pyruvate carboxylase supports the pulmonary tropism of metastatic breast

72. Rinaldi, G. et al. In vivo evidence for serine biosynthesis defined sensitivity of lung metastasis, but not of primar breast tumors, to mTORC1 inhibition. Mol. Cell https: doi.org/10.1016/j.molcel.2020.11.027 (2020).

73. Elia, I. et al. Proline metabolism supports metastasis formation and could be inhibited to selectively target metastasizing cancer cells. Nat. Commun. 8, 15267 (2017).

74. Diers, A. R., Broniowska, K. A., Chang, C. F. \& Hogg, N. Pyruvate fuels mitochondrial respiration and proliferation of breast cancer cells: effect of monocarboxylate transporter inhibition. Biochem. $J$. 444, 561-571 (2012).

75. Corbet, C. et al. Interruption of lactate uptake by inhibiting mitochondrial pyruvate transport unravels direct antitumor and radiosensitizing effects. Nat. Commun. 9, 1208 (2018).

76. Faubert, B. et al. Lactate metabolism in human lung tumors. Cell 171, 358-371.e9 (2017).

77. Elia, I., Schmieder, R., Christen, S. \& Fendt, S.-M. Organ-specific cancer metabolism and its potential for therapy. Handb. Exp. Pharmacol. 233, 321-353 (2016).

78. Yang, L. et al. Metabolic shifts toward glutamine regulate tumor growth, invasion and bioenergetics in ovarian cancer. Mol. Syst. Biol. 10, 728 (2014).

9. Rodrigues, M. F. et al. Enhanced OXPHOS, glutaminolysis and $\beta$-oxidation constitute the metastatic phenotype of melanoma cells. Biochem. $J$. 473, 703 (2016)

80. Xiang, L. et al. Glutaminase 1 expression in colorectal cancer cells is induced by hypoxia and required for tumor growth, invasion, and metastatic colonization. Cell Death Dis. 10, 40 (2019).

81. Du, F. et al. SOX12 promotes colorectal cancer cell proliferation and metastasis by regulating asparagin synthesis. Cell Death Dis. 10, 239 (2019).

82. Zhang, $C$. et al. Glutaminase 2 is a novel negative regulator of small GTPase Rac1 and mediates p53 function in suppressing metastasis. elife $\mathbf{5}$, e10727-e10727 (2016)

83. Kuo, T.-C. et al. Glutaminase 2 stabilizes Dicer to repress Snail and metastasis in hepatocellular carcinoma cells. Cancer Lett. 383, 282-294 (2016). 
84. Sugano, K. M. K., Ohtani, H., Nagahara, H., Shibutani, M. \& Hirakawa, K. Expression of $x C T$ as a predictor of disease recurrence in patients with color
Anticancer Res. 35, 677-682 (2015).

85. Dornier, E. et al. Glutaminolysis drives membrane trafficking to promote invasiveness of breast cancer cells. Nat. Commun. 8, 2255 (2017).

86. Yae, T. et al. Alternative splicing of CD 44 mRNA by ESRP1 enhances lung colonization of metastatic cancer cell. Nat. Commun. 3, 883 (2012).

87. Chen, R. S. et al. Disruption of $x C T$ inhibits cancer cell metastasis via the caveolin-1/ $\beta$-catenin pathway.

88. Jin $\mathrm{H}$. et al Serum metabolo

(2014 lymph node metastasis of esophageal squamous cell

89. Toyoshima, K. et al. Analysis of circulating tumor cells derived from advanced gastric cancer. Int. $J$. Cancer 137, 991-998 (2015).

90. Liu, G. et al. Glutamate dehydrogenase is a novel prognostic marker and predicts metastases in colorecta cancer patients. J. Transl Med. 13, 144 (2015).

91. Jin, L. et al. The PLAG1-GDH1 axis promotes anoikis resistance and tumor metastasis through CamKK2-AMPK signaling in LKB1-deficient lung cancer. Mol. Cell 69, 87-99.e7 (2018).

92. Wang, Q. et al. Targeting ASCT2-mediated glutamin uptake blocks prostate cancer growth and tumour development. J. Pathol. 236, 278-289 (2015).

3. Shelton, L. M., Huysentruyt, L. C. \& Seyfried, T. N. Clutamine targeting inhibits systemic metastasis in the VM-M3 murine tumor model. Int. J. Cancer 127, 2478-2485 (2010)

94. Lanzardo, S. et al. Immunotargeting of antigen $\mathrm{xCT}$ attenuates stem-like cell behavior and metastatic progression
(2016).

95. Gaschler, M. M. \& Stockwell, B. R. Lipid peroxidation in cell death. Biochem. Biophys. Res. Commun. 482,

96. Peck, B. \& Schulze, A. Lipid desaturation - the next step in targeting lipogenesis in cancer? FEBS J. 283,

97. Rohrig, F. \& Schulze, A. The multifaceted roles of fatty acid synthesis in cancer. Nat. Rev. Cancer 16

98. Chen, M. et al. An aberrant SREBP-dependent lipogenic program promotes metastatic prostate cancer. Nat. Genet. 50, 206-218 (2018).

99. Pandey, V., Vijayakumar, M. V., Ajay, A. K., Malvi, P. \& Bhat, M. K. Diet-induced obesity increases melanoma progression: involvement of Cav-1 and FASN. Int $J$ Cancer 130, 497-508 (2012).

100. Jiralerspong, S. \& Goodwin, P. J. Obesity and breast cancer prognosis: evidence, challenges, and opport

101. O'Flanagan, C. H. et al. Metabolic reprogramming underlies metastatic potential in an obesity-responsive murine model of metastatic triple negative breast cancer. NPJ Breast Cancer 3, 26 (2017).

102. Sant'Anna-Silva, A. C. B. et al. Metabolic profile of oral squamous carcinoma cell lines relies on a higher demand of lipid metabolism in metastatic cells. Front. Oncol. 8, 13 (2018).

03. Pascual, G. et al. Targeting metastasis-initiating cells through the fatty acid receptor CD36. Nature 541 41-45 (2017).

This article identifies CD36+ metastasis-initiating cells in human oral carcinoma and other human cancer types and shows that blocking CD36 inhibits

metastasis formation.
04. Antalis, C. J., Uchida, A., Buhman, K. K. \& Siddiqui, R. A. Migration of MDA-MB-231 breast cancer cells depends on the avallability of exogenous lipids and cholesterol esterifict

105. Nath, A. \& Chan, C. Genetic alterations in fatty acid transport and metabolism genes are associated with metastatic progression and poor prognosis of human metastatic progression and poor pro
cancers. Sci. Rep. 6, 18669 (2016).

106. Nath, A., Li, I., Roberts, L. R. \& Chan, C. Elevated free fatty acid uptake via CD36 promotes epithelialmesenchymal transition in hepatocellular carcinoma. Sci. Rep. 5, 14752 (2015).

107. Ladanyi, A. et al. Adipocyte-induced CD36 expression drives ovarian cancer progression and metastasis. Oncogene 37, 2285-2301 (2018).

108. Yang, P. et al. Dietary oleic acid-induced CD36 promotes cervical cancer cell growth and metastasis via up-regulation Src/ERK pathway. Cancer Lett. 438 76-85 (2018).
109. Zaoui, M. et al. Breast-associated adipocytes secretome induce fatty acid uptake and invasiveness in breast cancer cells via CD36 independently of bo density. Cancers 11, 2012 (2019).

110. Xu, W. H. et al. Elevated CD36 expression correlates with increased visceral adipose tissue and predicts poor prognosis in ccRCC patients. J. Cancer 10, 4522-4531 (2019).

111. Hale, J. S. et al. Cancer stem cell-specific scavenger receptor CD36 drives glioblastoma progression. Stem Cell 32, 1746-1758 (2014)

112. Wang, R. et al. Fatty-acid receptor CD36 functions as a hydrogen sulfide-targeted receptor with its Cys333-Cys272 disulfide bond serving as a specific molecular switch to accelerate gastric cancer molecular switch to accelerate gastric cancer
metastasis. EBioMedicine 45, 108-123 (2019).

113. Pan, J. et al. CD36 mediates palmitate acid-induced metastasis of gastric cancer via AKT/CSK-3 $3 / \beta$-catenin

114. Sp, N. et al. Nobiletin inhibits CD36-dependent tumor angiogenesis, migration, invasion, and sphere formation through the CD36/STAT3/NF-kB signaling axis. Nutrients 10, 772 (2018).

115. Casciano, J. C. et al. MYC regulates fatty acid metabolism through a multigenic program in claudin868-884 (2020).

116. Watt, M. J. et al. Suppressing fatty acid uptake has therapeutic effects in preclinical models of prostate cancer. Sci. Transl Med. 11, eaau5758 (2019).

17. Deng, M. et al. CD36 promotes the epithelialmesenchymal transition and metastasis in cervical
cancer by interacting with TGF- $\beta$. J. Trans/ Med. 17 , cancer by int

118. Gharpure, K. M. et al. FABP4 as a key determinant of metastatic potential of ovarian cancer. Nat. Commun. 9, $2923(2018)$

119. Kawaguchi, K. et al. High expression of fatty acidbinding protein 5 promotes cell growth and metastatic potential of colore
$190-199$ (2016).

120. Wu, G. et al. FABP5 is correlated with poor prognosis and promotes tumour cell growth and metastasis in clear cell renal cell carcinoma. Eur. J. Pharmacol. 862 172637 (2019).

121. Ohata, T. et al. Fatty acid-binding protein 5 function in hepatocellular carcinoma through induction of epithelial-mesenchymal transition Cancer Med 6 , 1049-1061 (2017).

122. Wang, W. et al. FABP5 correlates with poor prognosis and promotes tumor cell growth and metastasis in cervical cancer. Tumor Biol. 37, 14873-14883 (2016).

123. Pan, J., Dai, Q., Zhang, T. \& Li, C. Palmitate acid promotes gastric cancer metastasis via $\mathrm{FABP} / \mathrm{SP}$ UCA1 pathway. Cancer Cell Int. 19, 69 (2019). 124. Carbonetti, G. et al. FABP5 coordinates lipid signaling
that promotes prostate cancer metastasis. Sci. Rep. $\mathbf{9}$, 18944 (2019)

125. Ku, C.-Y., Liu, Y.-H., Lin, H.-Y., Lu, S.-C. \& Lin, J.-Y. Liver fatty acid-binding protein (L-FABP) promotes cellular angiogenesis and migration in hepatocellul carcinoma. Oncotarget 7, 18229-18246 (2016).

26. Zhang, M. et al. Adipocyte-derived lipids mediat melanoma progression via FATP proteins. Cancer Discov. 8, 1006-1025 (2018).

127. Seguin, F. et al. The fatty acid synthase inhibitor orlistat reduces experimental metastases and angiogenesis in B16-F10 melanomas. Br. J. Cancer 107, $977(2012)$

128. Zaytseva, Y. Y. et al. Inhibition of fatty acid synthase attenuates CD44-associated signaling and reduces
metastasis in colorectal cancer. Cancer Res. 72, 1504 (2012).

129. Jafari, N. et al. De novo fatty acid synthesis-drive sphingolipid metabolism promotes metastatic potential of colore

130. Vriens, K. et al. Evidence for an alternative fatty acid desaturation pathway increasing cancer plasticity. desaturation pathway increasing
Nature 566, 403-406 (2019).

131. Triki, M. et al. mTOR signaling and SREBP activity increase FADS2 expression and can activate sapienate increase FADS2 expression and can activate
biosynthesis. Cell Rep. 31, 107806 (2020).

132. Ran, H. et al. Stearoyl-CoA desaturase-1 promotes colorectal cancer metastasis in response to glucose by
suppressing PTEN. J. Exp. Clin. Cancer Res. 37, 54 (2018).

133. Zhao, J. et al. Exogenous lipids promote the growth 2105-2115 (2017).
134. Li, J. et al. Lipid desaturation is a metabolic marker and therapeutic target of ovarian cancer stem cells Cell 20 303-314.e5 (2017).

135. Vivas-García, Y et al. Lineage-restricted regulation of SCD and fatty acid saturation by MITF controls melanoma phenotypic plasticity. Mol. Cell 77 . 120-137.e9 (2020).

This work reveals that SCD1 is regulated by MITF being implicated in melanoma proliferation. SCD inhibition leads to endoplasmic reticulum stress formation.

136. Bellenghi, M. et al. SCD5-induced oleic acid production reduces melanoma malignancy by intracellular retention of SPARC and cathepsin B. J. Pathol. 236, 315-325 (2015).

137. Lee, H. J. et al. Cholesterol esterification inhibition suppresses prostate cancer metastasis by impairing (2018)

138. Li, J. et al. Abrogating cholesterol esterification suppresses growth and metastasis of pancreatic cancer. Oncogene 35, 6378-6388 (2016).

139. Bi, M. et al. Effect of inhibiting ACAT-1 expression on the growth and metastasis of Lewis lung carcinoma. Oncol. Lett. 18, 1548-1556 (2019).

140. Wang, Y. Y. et al. Mammary adipocytes stimulate breast cancer invasion through metabolic remodeling of tumor cells. JCI Insight 2, e87489 (2017).

141. Miranda, F. et al. Salt-inducible kinase 2 couples ovarian cancer cell metabolism with survival at the adipocyte-rich metastatic niche. Cancer Cell 30 273-289 (2016).

142. Xiong, Y. et al. CPT1 A regulates breast cancerassociated lymphangiogenesis via VEGF signaling. Biomed. Pharmacother. 106, 1-7 (2018).

143. Pucci, S. et al. Carnitine palmitoyl transferase-1A (CPT1A): a new tumor specific target in human
cancer. Oncotarget 7, 19982-19996 (2016).

144. Wang, C. et al. Elevated level of mitochondrial reactive oxygen species via fatty acid $\beta$-oxidation in cancer stem cells promotes cancer metastasis by inducin epithelial-mesenchymal transition. Sten

145. Comes A. P. et al Age-induced accumulation of methylmalonic acid promotes tumour progression. (2020).

This study shows that age-dependent metabolic change, that is, methylmalonic acid accumulation in the blood, promotes cancer progression by inducing Sox4.

146. Notarnicola, M. et al. Serum lipid profile in colorectal cancer patients with and without synchronous distant metastases. Oncology 68, 371-374 (2005).

147. Liu, Y.-L. et al. Association of serum lipid profile with distant metastasis in breast cancer patients.
Zhonghua Zhong Liu Za Zhi 34, 129-131 (2012).

48. Acharya, S., Rai, P., Hallikeri, K., Anehosur, V. \& Kale, J. Serum lipid profile in oral squamous cell carcinoma: alterations and association with some clinicopathological parameters and tobacco use. Int. J. Oral. Maxillofac. Surg. 45, 713-720 (2016).

149. Wang, Y. N. et al. CPT1A-mediated fatty acid oxidation promotes colorectal cancer cell metastasis by inhibiting anoikis. Oncogene 37, 6025-6040 (2018).

150. Jiang, L. et al. Reductive carboxylation supports redox homeostasis during anchorage-independent growth. Nature 532, 255-258 (2016)

151. Lignitto, L. et al. Nrf2 activation promotes lung cancer metastasis by inhibiting the degradation of Bach 1 . Cell 178, 316-329.e18 (2019).

152. Yu, G. et al. Organelle-derived acetyl-CoA promotes prostate cancer cell survival, migration, and metastasis via activation of calmodullin kinase

53. McCoy, F. et al. Metabolic activation of CaMKII by coenzyme A. Mol. Cell52 325-339 (2013).

pr. Un protects melanoma cells from ferroptosis. Nature
$158,113-118(2020)$. This work reveals that metastasizing melanoma cells in lymphatic vessels experience less oxidative stress than cells in the blood because they are

efficient to form metastases.
. of cervical cancer by reprogramming fatty acid metabolism. Cancer Res. 78, 877 (2018).

156. Mukherjee, A. et al. Adipocyte-induced FABP expression in ovarian cancer cells promotes metastasis and mediates carboplatin resistance. Cancer Res. 80, 1748-1761 (2020). 
157. Jiang, L. et al. Up-regulated FASN expression promotes transcoelomic metastasis of ovarian
cancer cell through epithelial-mesenchymal transition. cancer cell through epithelial-mesenchym
Int. J. Mol. Sci. 15, 11539-11554 (2014)

158. Li, J. et al. CD147 reprograms fatty acid metabolism in hepatocellular carcinoma cells through Akt/mTOR/ SREBP1C and P38/PPAP

59. Liu $C$. colonization through upregulation of stearoyl-COA desaturase 1 in tumor cells. Oncogene 37 1519-1533 (2018).

160. Blomme, A. et al. Myoferlin regulates cellular lipid metabolism and promotes metastases in triple-

negative breast cancer. Oncogene 36, 2116 (2016).

61. Lee, C.-K. et al. Tumor metastasis to lymph nodes requires YAP-dependent metabolic adaptation.

Science 363, 644 (2019).

62. Lu, J. The Warburg metabolism fuels tumor metastasis. Cancer Metastasis Rev. 38, 157-164 (2019).

163. Lehuédé, C., Dupuy, F., Rabinovitch, R., Jones, R. G. \& Siegel, P. M. Metabolic plasticity as a determinant of (2016).

164. Lu, M. et al. ACOT12-dependent alteration of acetylCOA drives hepatocellular carcinoma metastasis by epigenetic induction of epithelial-mesenchymal

65. Sun, L. et al. Decreased expression of actyl-CoA synthase 2 promotes metastasis and predicts poo prognosis in hepatocellular carcinoma. Cancer Sci. 108, 1338-1346 (2017).

166. Comerford, S. A. et al. Acetate dependence of tumors Cell 159, 1591-1602 (2014)

167. Schug, Z. T. et al. Acetyl-CoA synthetase 2 promotes acetate utilization and maintains cancer cell growth

under metabolic stress. Cancer Cell 27, 57-71 (2015) for human glioblastoma and brain metastases. Cell 159, 1603-1614 (2014). 169. Pollari, S. et al. Enhanced serine production by
bone metastatic breast cancer cells stimulates osteoclastogenesis. Breast Cancer Res. Treat. 125 421-430 (2011).

170. Zhu J. et al. High expression of PHGDH predicts poor prognosis in non-small cell lung cancer. Transl Oncol. 9, 592-599 (2016).

171. Song, Z., Feng, C., Lu, Y., Lin, Y. \& Dong, C. PHGDH is an independent prognosis marker and contributes cell proliferation, migration and invasion in human

pancreatic cancer. Gene 642, 43-50 (2018).

2. Kim, H. M., Jung, W. H. \& Koo, J. S. Site-specific metabolic phenotypes in metastatic breast cancer

173. Samanta, D. et al. PHGDH expression is required for mitochond ial redox homeostasis, breast cancer stem 76, 4430 (2016).

174. Ngo, B. et al. Limited environmental serine and glycine confer brain metastasis sensitivity to PHGDH inhibition. Cancer Discov. 12, 1352-1373 (2020).

175. Cao, Y. et al. Glutamic pyruvate transaminase GPT promotes tumorigenesis of breast cancer cells by activating sonic hedgehog signaling. Theranostics 7 , 3021-3033 (2017).

176. Pavlova, N. N. et al. As extracellular glutamine levels decline, asparagine becomes an essential amino acid. Cell Metab. 27, 428-438.e5 (2018)

177. Knott, S. R. V. et al. Asparagine bioavailability govern metastasis in a model of breast cancer. Nature 554 , 378 (2018).

This work finds limiting asparagine bioavailability by dietary asNS or treatment with L-asparaginase, or dietary asparagine restriction, reduces metastasis

78. Tanner, J J. Fendt. S.-M \& Becker. D. F. The proline cycle as a potential cancer therapy target.
Biochemistry 57, 3433-3444 (2018).
179. Loayza-Puch, F. et al. Tumour-specific proline vulnerability uncovered by differential riboso

180. Wang, D. et al. PYCR1 promotes the progression of regulation of miR-488. Biomed. Pharmacother. 111,

181. Fang, E. et al. Therapeutic targeting of MZF1-AS1/ PARP1/E2F1 axis inhibits proline synthesis and (2019)

182. Sahu, N. et al. Proline starvation induces unresolved ER stress and hinders mTORC1-dependent tumorigenesis. Cell Metab. 24, 753-761 (2016).

83. Fendt, S.-M. Metabolic vulnerabilities of metastasizing cancer cells. BMC Biol. 17, 54 (2019).

84. Lunt, S. Y. \& Fendt, S.-M. Metabolism cornerstone of cancer initiation, progression, immun evasion and treatment

185. Wang, Y.-n. et al. CPT1A-mediated fatty acid oxidation promotes colorectal cancer cell metastasis (2018).

186. Kamarajugadda, S. et al. Glucose oxidation modulates anoikis and tumor metastasis. Mol. Cell. Biol. 32 1893-1907 (2012).

187. Kruse, N. J. \& Bornstein, P. The metabolic requirements for transcellular movement and
secretion of collagen. J. Biol. Chem. 250, 4841-4847 (1975)

88. Park, J. H. et al. Fatty acid oxidation-driven Src links mitochondrial energy reprogramming and oncogenic properties in triple-negative breast cancer. Cell Rep. 14, 2154-2165 (2016)

189. Wright, H. J. et al. CDCP1 drives triple-negative breast cancer metastasis through reduction of lipid-droplet abundance and stimulation of fatty acid oxidation.

20. Andrzejewski. S. et al. PCC-1 .

cancer metastasis and confers bioenergetic flexibility cancer metastasis and confers bioenergetic flexibillty (2017).

191. Dupuy, F. et al. PDK1-dependent metabolic reprogramming dictates metastatic potential in breast cancer. Cell Metab. 22, 577-589 (2015).

Loo, J. M. et al. Extracellular metabolic energetics (2015).

193. Payne, C. E. et al. A novel selective and orally bioavailable Nav 1.8 channel blocker, PF-01247324 attenuates nociception and sensory neuron excitability. Br. J. Pharmacol. 172, 2654-2670 (2015)

194. Fernandes, M., Rosel, D. \& Brábek, J. Solid cancer: hew tumour spread endpoint opens nove

195. Chaika N V. et a . Differential expression (2019). genes in tumor and stromal composs of primary and metastatic loci in pancreatic adenocarcinoma. PLOS ONE 7, e32996 (2012).

196. Davis, R. T. et al. Transcriptional diversity and bioenergetic shift in human breast cancer metastasis revealed by single-cell RNA sequencing. Nat. Cell Biol. 22, 310-320 (2020)

197. Karaayvaz, M. et al. Unravelling subclona heterogeneity and aggressive disease states in TNBC through single-cell RNA-seq. Nat. Commun. 9, 3588 (2018)

198. Basnet, H. et al. Flura-seq identifies organ-specific metabolic adaptations during early me
colonization. elife 8, e43627 (2019).

199. Chen, E. I. et al. Adaptation of energy metabolism in breast cancer brain metastases. Cancer Res. 67 1472 (2007).

200. Fischer, G. M. et al. Molecular profiling reveals unique immune and metabolic features of melanoma brain
metastases. Cancer Discov. 9, 628-645 (2019).
201. Davidson, S. M. et al. Environment impacts the metabolic dependencies of Ras-driven non-small cell lung cancer. Cell Metab. 23,517-528 (2016).

202. Hensley, C. T. et all Metabolich he .

Metabolism of [U-13 C]glucose in human brain tumors in

204. Fernández-García, J., Altea-Manzano, P., Pranzini, E. $\ltimes$ Fendt, S.-M. Stable isotopes for tracing mammaliancell metabolism in vivo. Trends Biochem. Sci. 45. 185-201 (2020).

205. Buescher, J. M. et al. A roadmap for interpreting ${ }^{3} \mathrm{C}$ metabolite labeling patterns from cells. Curr. Opin. Biotechnol. 34, 189-201 (2015).

206. Fong, M. Y. et al. Breast-cancer-secreted miR-122 reprograms glucose metabolism in premetastatic niche to promote metastasis. Nat. Cell Biol. 17 183-194 (2015).

207. Hao, Y. et al. Investigation of lipid metabolism dysregulation and the effects on immune multiple omics (2019).

8. Bestron Nat. Med. 20, 1327 (2014).

209. Wang $\mathrm{H}$. et al. CD36-mediated metabolic adaptation supports regulatory $\mathrm{T}$ cell survival and function in tumors. Nat. Immunol. 21, 298-308 (2020).

10. Su, P. et al. Enhanced lipid accumulation and metabolism are required for the differentiation and activation of tumor-associated macrophages. Cancer Res. 80, 1438-1450 (2020)

211. Niavarani, S. R. et al. Lipid accumulation impairs natural killer cell cytotoxicity and tumor control in the

postoperative period. $B M C$ Cancer $19,823(2019)$.
Michelet, X. et al. Metabolic reprogramming of natura killer cells in obesity limits antitumor responses.
kitus Nat. Immunol. 19, 1330-1340 (2018).

213. Pan, Y. et al. Survival of tissue-resident memory T cells requires exogenous lipid uptake and metabolism. Nature 543, 252 (2017).

214. García-Mulero, S. et al. Lung metastases share common immune features regardless of primary tumor origin. J. Immunother. Cancer 8, e000491 (2020).

Acknowledgements

The authors regret the inability to cite all studies that have shaped the understanding of cancer metastasis metabolism. S.-M.F. acknowledges funding from the European Research Council under the ERC Consolidator Grant Agreement n. 771486 - MetaRegulation, FWO - Odysseus II, FWO research projects (G098120N, G088318N), KU Leuven Methusalem Co-funding and Fonds Baillet Latour. C.B. (Stichting tegen Kanker (STK 1303), the Flemish gor society FWO (COA0818N) and the National Institutes of Health (NIH)/National Cancer Institute (NCI) (R01CA201537).

Author contributions

Both authors contributed equally to all aspects of the article.

Competing interests

S.-M.F. has received funding from Bayer, Merck and BlackBelt Therapeutics and has consulted for Fund+. G.B. declares no competing interests. 